\title{
Paper Enhancement of Air-ground Matching by Means of a Chirped Multilayer Structure: Electromagnetic Modeling with the Method of Single Expression
}

\author{
Hovik Baghdasaryan $^{1}$, Tamara Knyazyan ${ }^{1}$, Tamara Hovhannisyan ${ }^{1}$, \\ Marian Marciniak ${ }^{2}$, and Lara Pajewski ${ }^{3}$ \\ ${ }^{1}$ National Polytechnic University of Armenia, Yerevan, Armenia \\ ${ }^{2}$ National Institute of Telecommunications, Warsaw, Poland \\ ${ }^{3}$ Sapienza University of Rome, Department of Information Engineering, Electronics and Telecommunications, Rome, Italy
}

https://doi.org/10.26636/jtit.2017.121117

\begin{abstract}
The enhancement of air-ground electromagnetic matching by means of a chirped multilayer structure is investigated. The modeling and simulation of the considered structure are performed by using the method of single expression (MSE), which is a convenient and accurate tool for wavelengthscale simulations of multilayers comprising lossy, amplifying or nonlinear (Kerr-type) materials. Numerical results show that a suitable chirped multilayer structure can reduce the reflection from the ground. Different values of the number of layers and of the layer thicknesses are considered. The distributions of the electric field components and the power flow density within the modelled structures are calculated.
\end{abstract}

Keywords-chirped multilayer structure, ground matching, method of single expression.

\section{Introduction}

Ground Penetrating Radar (GPR) is an electromagnetic technique currently in use for detection and imaging of buried objects, with resolution ranging from centimeters to few meters depending on the operation wavelength of the radar antenna [1]-[6]. The principles of GPR for detecting buried objects are well known, indeed GPR uses the same fundamental physical principles as conventional radars. GPR is a technique typically operating at frequencies included within the $10 \mathrm{MHz}$ to $5 \mathrm{GHz}$ range. The chosen frequency band depends on measurement purposes and properties of the investigated media. Target objects can be buried pipes, cables and reinforcing elements, caverns, flaws and cracks, as well as ground water and moisture, and more [6]-[11]. Nowadays, GPR is widely applied in archaeological investigations and for cultural heritage management, planetary exploration, forensics, civil, hydrogeological, geophysical and geotechnical engineering [6]-[11].

GPR detects the electromagnetic field backscattered by the targets and generates an image of it, which is interpreted after proper signal processing. The GPR transmitter emits electromagnetic waves towards the ground or structure under test. When the waves encounter a buried object or a boundary between materials having different permittivity and conductivity values, they are reflected, refracted and scattered back to the radar. A receiving antenna catches the returned signal, which is analyzed and recorded. Depth and shape of the objects are calculated from the travel time and amplitude of the reflected signals, along an acquisition profile.

The signal attenuation and penetration depth at the desired operating frequency are the main factors influencing the functionality and resolution of a GPR device [2]-[6]. Resolution of GPR primarily is a function of the radar frequency and the permittivity of the detected medium. High resolution can be reached at the cost of penetration depth, because the attenuation of electromagnetic waves in common ground materials, as well as in materials utilized in manmade structures, is rather high and increases with frequency. At a lower frequency, GPR can sample deeper, but the resolution is lower. A higher frequency range gives a narrower pulse, yielding a higher time or depth resolution. On the other hand, attenuation increases with frequency. Therefore, a high frequency signal cannot propagate far and the depth of detection becomes shallower [12], [13]. To give a practical example, for applications requiring high resolution and a penetration depth down to approximately $60 \mathrm{~cm}$, such as assessment of concrete structures, a frequency spectrum centered over $1 \mathrm{GHz}$ is normally used. For applications requiring penetration from tens of centimeters to hundreds of meters, frequencies between $12.5 \mathrm{MHz}$ to $500 \mathrm{MHz}$ are used.

Antennas are essential components of GPR systems, which transmit and receive electromagnetic waves. Various types of antennas can be used: dipole, bowtie and horn antennas are the most common. Several systems use two separate antennas for transmitting and receiving, although they can 
be packaged together. Some commercial systems employ shielded antennas to avoid reflections from objects in the air. The antenna gain is very important for efficiently emitting and receiving the electromagnetic energy. Antennas with a high gain improve the signal/noise ratio. A lower operating frequency requires larger antennas. Small antennas make the system compact, but they have a low gain at lower frequencies [12], [13].

Though the GPR technique is mature enough, some problems are still waiting for their solution [6]. One of them is to achieve a better matching of the electromagnetic waves emitted by the GPR with the ground, which would increase the signal penetration depth, minimize back reflections at the air-ground interface and enhance the signal/noise ratio at the receiving end. Different antenna configurations and antenna-medium interactions have been studied in the literature, mostly by using the finite-difference time-domain (FDTD) method or the method of moments [14]-[16].

In this paper, the possibility to enhance air-ground electromagnetic matching by means of a suitable chirped multilayer structure is studied. The wavelength-scale electromagnetic modeling and simulation of a chirped multilayer structure over a dielectric half-space, illuminated by a monochromatic plane wave, is performed by using the method of single expression (MSE) [17]-[22] (Section 2). The behavior of different structures is analyzed, to find advantageous configurations for matching enhancement (Section 3). Preliminary results were presented in [22]. Further studies are necessary, to consider more realistic sources and ultra-wideband radiation.

\section{Electromagnetic-modeling Method}

The method of single expression (MSE) allows to accurately analyze wavelength-scale multilayer structures comprising lossy, amplifying or nonlinear (Kerr-type) dielectric, semiconductor or metallic materials [17]-[22]. In this Section, the backbone of the MSE for normal wave incidence on a multilayer structure is resumed.

From Maxwell's equations in the one-dimensional case, the Helmholtz equation can be obtained, for the linearly polarized complex electric field component $\dot{E}_{x}(z)$ :

$$
\frac{d^{2} \dot{E}_{x}(z)}{d z^{2}}+k_{0}^{2} \dot{\varepsilon}(z) \dot{E}_{x}(z)=0,
$$

where $k_{0}=\frac{\omega}{c}$ is the free space propagation constant and $\dot{\varepsilon}(z)=\varepsilon^{\prime}(z)+j \varepsilon^{\prime \prime}(z)$ is the complex permittivity of a medium.

The essence of the MSE is the use of a general solution of the Helmholtz equation, having the following single expression:

$$
\dot{E}_{x}(z)=U(z) \cdot \mathrm{e}^{-j S(z)}
$$

instead of the traditional solution expressed as a sum of counter-propagating waves. In (2), $U(z)$ and $S(z)$ are real quantities describing the electric field amplitude and phase, respectively. Time dependence $\mathrm{e}^{j \omega t}$ is assumed, but suppressed in the formulas. The expression (2) can de- scribe all the possible electric field amplitude distributions in a medium, corresponding to propagating, standing and evanescent waves. This gives advantages in the investigation of the interaction of electromagnetic waves with longitudinally non-uniform linear media and with intensity dependent non-linear media.

By introducing (2) in (1), the Helmholtz equation can be rewritten as a set of first-order differential equations:

$$
\left\{\begin{array}{l}
\frac{d U(z)}{d\left(k_{0} z\right)}=Y(z) \\
\frac{d Y(z)}{d\left(k_{0} z\right)}=\frac{P^{2}(z)}{U^{3}(z)}-\varepsilon^{\prime}(z) \cdot U(z) \\
\frac{d P(z)}{d\left(k_{0} z\right)}=\varepsilon^{\prime \prime}(z) \cdot U^{2}(z)
\end{array}\right.
$$

where $Y(z)$ is the spatial derivative of $U(z)$ and $P(z)$ is a quantity that is proportional to the power flow density (Poynting vector) in a medium, i.e. $P(z)=U^{2}(z) \frac{d S(z)}{d\left(k_{0} z\right)}$. The sign of $\varepsilon^{\prime}(z)$ can assume either positive or negative values, describing relevant electromagnetic features of dielectric materials or plasma, respectively. The sign of $\varepsilon^{\prime \prime}(z)$ indicates loss or gain in a medium.

The set of equations in (3) can be integrated numerically starting from the non-illuminated side of a multilayer structure, where only one outgoing traveling wave is supposed to be present. The initial values for the integration can be obtained from the boundary conditions of electrodynamics at the non-illuminated side of the structure $(z=L)$, i.e., $U(L)=E_{t r}, Y(L)=0$, and $P(L)=\sqrt{\varepsilon_{r}} E_{t r}^{2}$, where $E_{t r}$ and $\varepsilon_{r}$ are the amplitude of the transmitted wave and the permittivity of the medium beyond the multilayer structure at $z>L$, respectively. Numerical integration of (3) continues step by step towards the illuminated side of the structure, taking into account the actual value of the permittivity for the given coordinates at each step of integration. In the process of integration, any variable in (3) can be calculated and recorded, so that it is possible to obtain full information regarding the distribution of the electric field amplitude in the space, as well as the distribution of its derivative and power flow density.

At the interfaces between different material of the multilayer structure, the ordinary boundary conditions of electrodynamics bring to the continuity of $U(z), Y(z)$ and $P(z)$. From the boundary conditions of electrodynamics at the illuminated side of the structure $(z=0)$, the amplitude of the incident wave $E_{\text {inc }}$ :

$$
E_{i n c}=\left|\frac{U^{2}(0) \cdot \sqrt{\varepsilon_{l}}+P(0)+j U(0) \cdot Y(0)}{2 U(0) \cdot \sqrt{\varepsilon_{l}}}\right|,
$$

and the power reflection coefficient $R$ :

$$
R=\left|\frac{E_{r e f}}{E_{\text {inc }}}\right|^{2}=\left|\frac{U^{2}(0) \cdot \sqrt{\varepsilon_{l}}-P(0)-j U(0) \cdot Y(0)}{U^{2}(0) \cdot \sqrt{\varepsilon_{l}}+P(0)+j U(0) \cdot Y(0)}\right|^{2}
$$

are restored at the end of the calculation. Here, $U(0)$ is the amplitude of the electromagnetic wave, $Y(0)$ is its derivative and $P(0)$ the power flow density at the illuminated 
interface of the structure $z=0, E_{\text {ref }}$ is the amplitude of the reflected wave, and finally $\varepsilon_{l}$ is the permittivity of the medium in front of the structure, for $z<0$.

The power transmission coefficient $T=\frac{E_{t r}^{2} \sqrt{\varepsilon_{r}}}{E_{i n c}^{2} \sqrt{\varepsilon_{l}}}$ is defined as the ratio of transmitted power to the incident one.

\section{Numerical Results}

The geometry of the modeled and simulated structure is represented in Fig. 1.

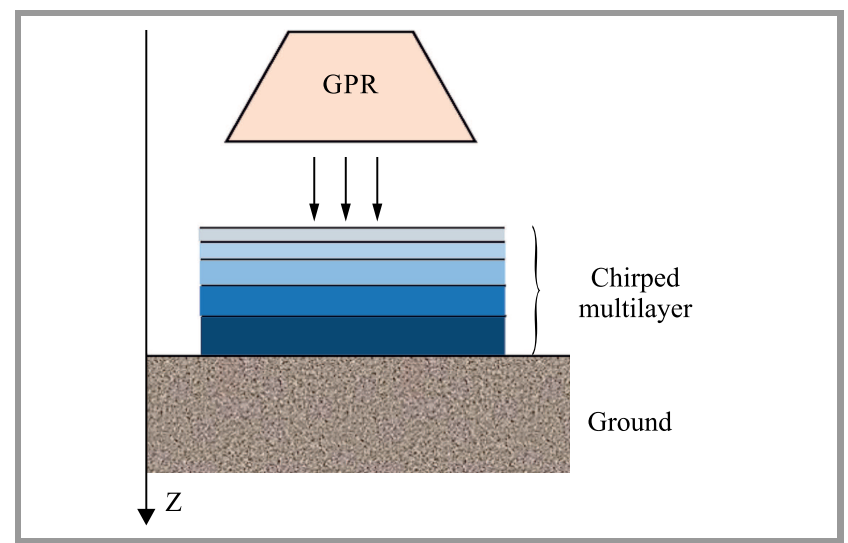

Fig. 1. Plane wave interaction with the ground through a chirped multilayer structure.

Normal incidence of a plane wave with wavelength $\lambda_{0}=$ $10 \mathrm{~cm}$ on a ground of complex permittivity $\dot{\varepsilon}_{g r}=\varepsilon_{g r}^{\prime}+$ $j \varepsilon_{g r}^{\prime \prime}=10-j 0.3[23]$ is considered. A chirped multilayer structure is positioned above the ground. It consists of two alternating dielectric slabs of low $\varepsilon_{\text {low }}^{\prime}=2.25$ and high $\varepsilon_{\text {high }}^{\prime}=6.0$ permittivity creating bilayers of increasing thickness towards the ground. A numerical analysis and optimization carried out by using the MSE revealed that bilayers should have the following normalized thicknesses (NT): $N T_{i}=0.3471 ; 0.4471 ; 0.5471$, where $N T_{i}=\frac{L_{i}}{\lambda}(i=1,2,3$ is a number of bilayers) starting from the illuminated side, being $\lambda=\frac{\lambda_{0}}{\sqrt{\varepsilon}}$ the wavelength in the slab with $\varepsilon=\varepsilon_{\text {low }}^{\prime}$ and $\varepsilon=\varepsilon_{\text {high }}^{\prime}$ permittivity, respectively.

Results for two particular structures are presented in the following. The permittivity profile of such structures is plotted in Fig. 2. For the first structure (Fig. 2a):

$N T_{1}=0.3471\left(\varepsilon_{\text {high }}^{\prime}\right.$ and $\left.\varepsilon_{\text {low }}^{\prime}\right)$,

$N T_{2}=0.4471\left(\varepsilon_{\text {high }}^{\prime}\right.$ and $\left.\varepsilon_{\text {low }}^{\prime}\right)$,

$N T_{3}=0.5471\left(\varepsilon_{\text {high }}^{\prime}\right.$ and $\left.\varepsilon_{\text {low }}^{\prime}\right)$.

The material with low permittivity $\varepsilon_{\text {low }}^{\prime}$ is adjacent to the ground.

For the second structure (Fig. 2b):

$N T_{1}=0.3471\left(\varepsilon_{\text {low }}^{\prime}\right.$ and $\left.\varepsilon_{\text {high }}^{\prime}\right)$,

$N T_{2}=0.4471\left(\varepsilon_{\text {low }}^{\prime}\right.$ and $\left.\varepsilon_{\text {high }}^{\prime}\right)$,

$N T_{3}=0.5471\left(\varepsilon_{\text {low }}^{\prime}\right.$ and $\left.\varepsilon_{\text {high }}^{\prime}\right)$.

The material with high permittivity $\varepsilon_{\text {high }}^{\prime}$ is adjacent to the ground. (a)

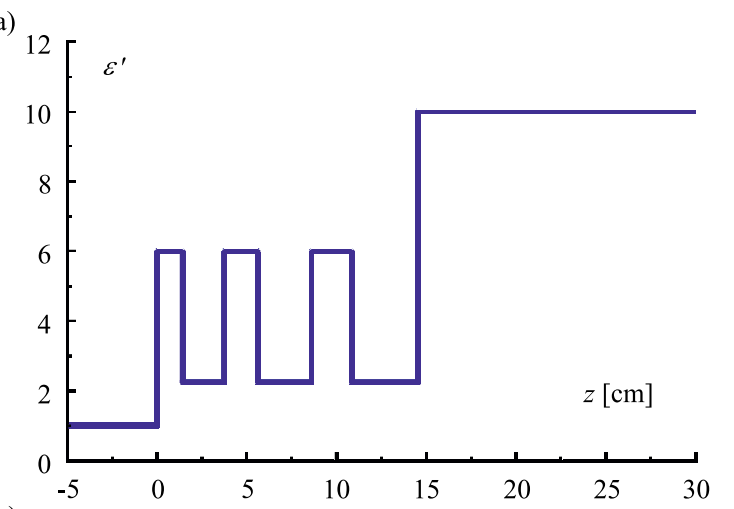

(b)

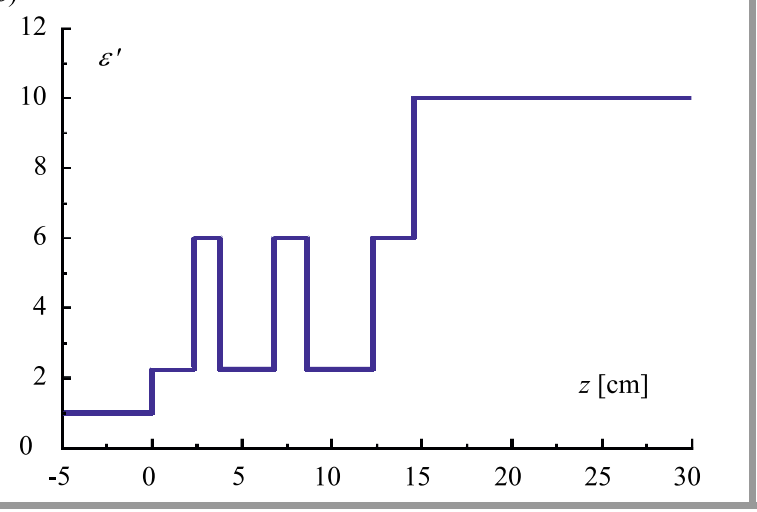

Fig. 2. Permittivity profiles of two chirped multilayer structures positioned on the ground. Wave incidence from the left side of the graphs is considered.

The reflectance $R$ from the structures air-multilayer-ground or air-ground interface is defined as $R=\frac{P_{\text {ref }}}{P_{\text {inc }}}$ - see Eq. (5), where $P_{i n c}$ is a power flow density (Poynting vector) of the electromagnetic wave impinging on the multilayer structure, and $P_{i n c}$ is a power flow density (Poynting vector) of the electromagnetic wave reflected from the structure.

In the absence of a chirped multilayer structure the reflectance $R_{g r}$ from the ground is defined as:

$$
R=\left|\frac{\sqrt{\varepsilon_{l}}-\sqrt{\left|\dot{\varepsilon}_{g r}\right|}}{\sqrt{\varepsilon_{l}}+\sqrt{\left|\dot{\varepsilon}_{g r}\right|} \mid}\right|^{2},
$$

which is a reflectance from the air-ground interface, the so-called Fresnel reflection coefficient. For the considered case, in the absence of the chirped multilayer structure, the reflectance $R_{g r}$ from the air-ground interface equals to $R_{g r} \approx 0.27$. This value has been calculated by using the MSE and the formula in Eq. (6).

The structure in Fig. 2b, where the layer of high permittivity is contacted with the ground, permits to attain airground matching with overall reflectance of $R \approx 0.0019$ while the structure in Fig. 2 a indicated reflectance $R \approx 0.25$ that is close to relevant value of reflectance $R_{g r} \approx 0.27$ for the air-ground interface (without multilayer structure). Thus, an enhancement of air-ground matching is obtained for the structure where the slab of $\varepsilon_{\text {high }}^{\prime}$ is contacted with the ground (Fig. 2b). 
In Fig. 3 the reflectance spectra of the air-ground and airmultilayer-ground for the two types of chirped multilayer structures are presented for the wavelength range of $\lambda_{0}=$ $(9.5 \div 10.5) \mathrm{cm}$.

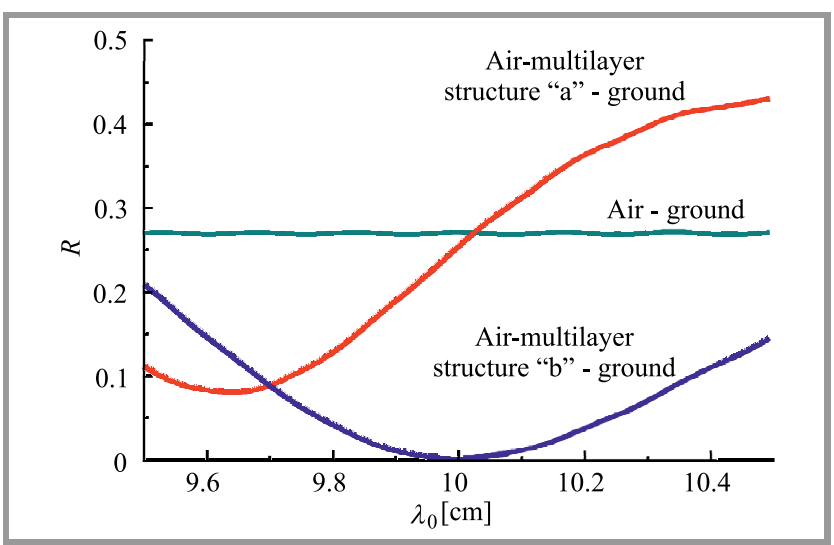

Fig. 3. Reflectance spectra of the air-ground and air-multilayerground for two types of chirped multilayer structures. (See color pictures online at www.nit.eu/publications/journal-jtit)

The chirped multilayer structure with a layer of high permittivity contacted with the ground, i.e. the structure " $b$ " in Fig. 2 has the lowest reflectance in the whole wavelength range as compared with the structure "a" in Fig. 2 and air-ground case. The lowest value of the reflectance $R \approx 0.0019$ of the structure " $\mathrm{b}$ " is observed at the wavelength $\lambda_{0}=10 \mathrm{~cm}$.

Thus, for better air-ground matching a chirped multilayer structure with a layer of high permittivity contacted with the ground (with reflectance of $R \approx 0.0019$ ) at the wavelength of $10 \mathrm{~cm}$ should be chosen (structure "b", Fig. 2b). Low reflectance permits deeper penetration of electromagnetic waves in the ground as it will be seen from the physics of difference of operation of two chirped multilayer structures, as well as of the air-ground interface in the absence of the multilayer structure.

In the absence of the multilayer structure, partially-standing wave amplitude of electric field is formed at the air-ground interface at $z<0$, which indicates essential reflectance from

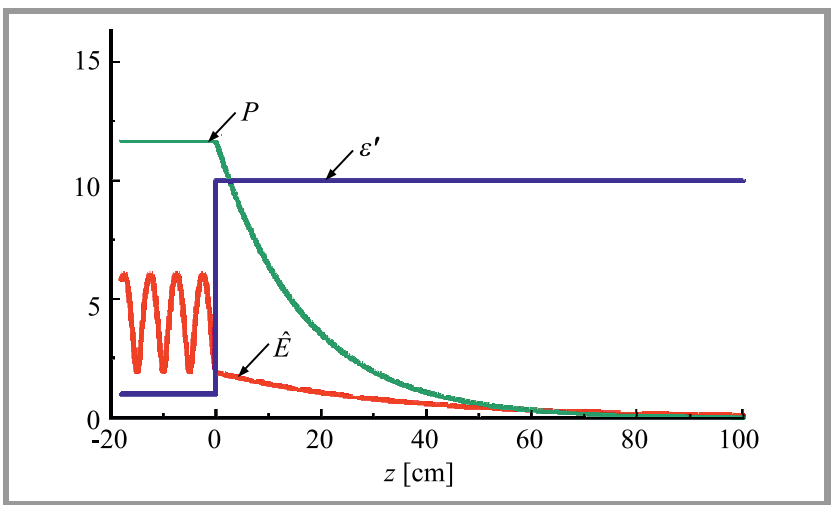

Fig. 4. Permittivity profile, distributions of electric field amplitude $\hat{E}$ and Poynting vector $P$ within the air and the ground. The amplitude of incident wave (from the left at $z<0$ ) is $E_{\text {inc }}=4$ a.u., $\lambda_{0}=10 \mathrm{~cm}, R_{g r} \approx 0.27$. the interface (Fig. 4). Exponentially decreasing electric field amplitude due to the loss in the ground is observed.

For $z<0$, the resultant Poynting vector $P$ is $P=P_{\text {inc }}-$ $P_{\text {ref }}=P_{\text {inc }}\left(1-R_{g r}\right)$. The Poynting vector $P$ is a constant value in the air and exponentially decreases in the ground. Consequently, because of the reflection from the air-ground interface and small resultant value of $P \approx 11.68$, the penetration depth into the ground is limited.

(a)

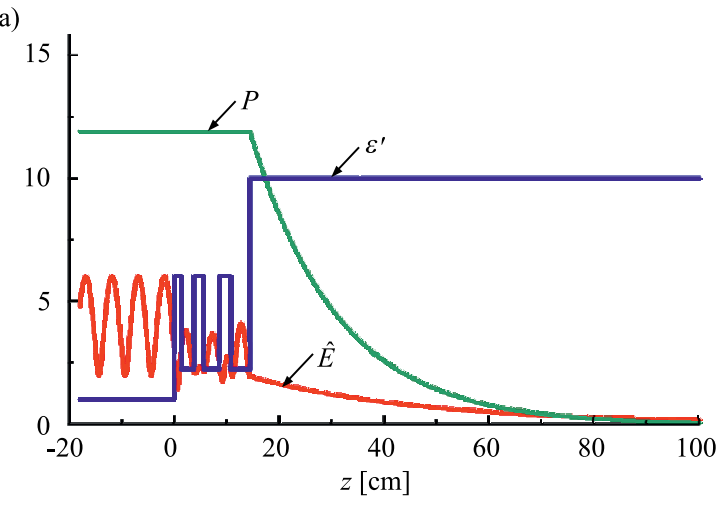

(b)

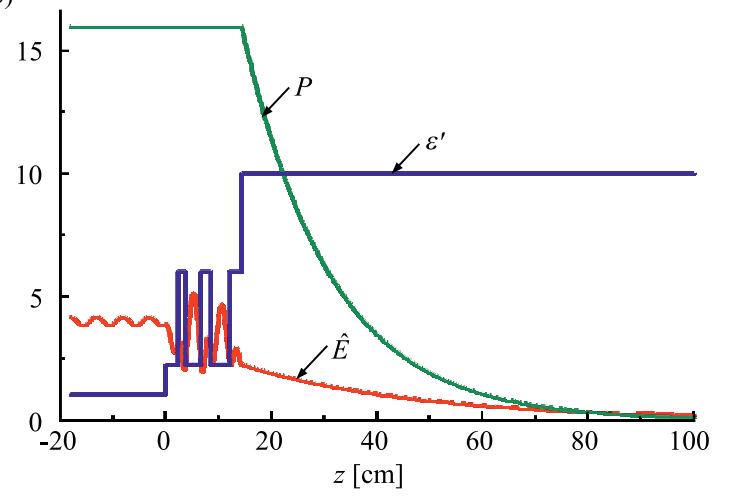

Fig. 5. Permittivity profile, distribution of electric field amplitude $\hat{E}$ and Poynting vector $P$ within and outside of the chirped multilayer structures. The amplitude of incident wave (from the left at $z<0)$ in both cases is the same $E_{\text {inc }}=4$ a.u. at $\lambda_{0}=10 \mathrm{~cm}$, $R \approx 0.25$ for the structure "a" and $R \approx 0.0019$ for the structure " $\mathrm{b}$ ".

The distributions of the electric field amplitude $\hat{E}$ and of the Poynting vector $P$ strongly depend on the alternation of layers in the chirped multilayer structure. The relevant distributions of electric field amplitude and Poynting vector within and outside the structures are presented in Fig. 5. Due to the absence of losses in the chirped multilayer structures, the Poynting vector $P$ is constant in them and the electric field amplitude $\hat{E}$ has an oscillating behavior following the permittivity profiles of multilayer structures. A partially-standing wave amplitude of the electric field is formed in front of the structure in Fig. 5a, indicating higher reflectance $R$ and $P \approx 11.93$. An almost traveling wave pattern is created in front of the structure in Fig. $5 \mathrm{~b}$, indicating lower reflectance $R$ and $P \approx 15.97$. The power flow penetrating into the ground increases of approximately 1.34 times, which causes a deeper penetration. 


\section{Conclusions}

A wavelength-scale electromagnetic analysis by means of the method of single expression (MSE) permitted to find chirped multilayer structures providing enhancement of airground matching, when the electromagnetic source is a monochromatic plane wave. The number and thicknesses of the layers can be optimized to minimize back reflection towards the electromagnetic source. The use of non-uniform (chirped) multilayers can enlarge the reflectance spectrum if compared to the standard quarter-wavelength multilayers. Interesting results have been found for a structure consisting of three bilayers of high and low permittivity, where the highest value of the permittivity does not exceed the permittivity of the ground. Bilayers have increasing thickness towards the ground and a better matching is achieved when the layer closer to the ground has the high permittivity.

The reflectance spectra and the distributions of electric field amplitude and Poynting vector within and outside the structures have been calculated. This allowed finding the favorable structures.

Mainly, the novelty of this work is in the application of a non-traditional method, the MSE, to the analysis of multilayers; the methods permits an easy calculation of the field amplitude and Poynting vector spatial distributions inside and outside the multilayer and in the presence of lossy media. Further studies are necessary, to design structures providing good results when illuminated by a pulsed electromagnetic wave.

\section{Acknowledgement}

This work originated from a Short-Term Scientific Mission funded by the COST Action TU1208 "Civil Engineering Applications of Ground Penetrating Radar", no. STSMTU1208-010816-080185. The authors are thankful to the members of the COST Action TU1208 for fruitful discussions.

\section{References}

[1] L. B. Conyers and D. Goodman, Ground Penetrating Radar: An Introduction for Archaeologists. London and New Delhi: AltaMira Press, Walnut Creek, 1997.

[2] D. J. Daniels, Ed., Ground Penetrating Radar, 2nd ed. London: The Institution of Engineering and Technology, 2004.

[3] H. M. Jol, Ground Penetrating Radar: Theory and Applications. Elsevier, 2009.

[4] D. J. Daniels, "GPR Design Challenges, COST Action TU1208, in Proc. The 1st Action's Gen. Meet. of the COST Action TU1208, Rome, Italy, 2013.

[5] R. Persico, Introduction to Ground Penetrating Radar. Wiley-IEEE Press, 2014.

[6] A. Benedetto and L. Pajewski, Civil Engineering Applications of Ground Penetrating Radar. Springer, 2015.

[7] F. Finck, "Introduction of a ground penetrating radar system for investigations on concrete structures", Otto-Graf-Journal, vol. 14, pp. 35-44, 2003.

[8] R. J. Yelf, "Application of Ground Penetrating Radar to civil and geotechnical engineering", Electromag. Phenom., vol. 7, no. 18, pp. 102-117, 2007.

[9] D. Goodman and S. Piro, GPR Remote Sensing in Archaeology. Berlin Heidelberg: Springer, 2013.

[10] L. Pajewski and A. Benedetto, Eds., Civil Engineering Applications of Ground Penetrating Radar, Proceedings of COST Action TU1208: First General Meeting, Rome, Italy, 2013.

[11] L. Pajewski and X. Derobert, Eds., Proceedings of COST Action TU1208: Civil Engineering Applications of Ground Penetrating Radar, 2014 Working Group Progress Meeting, Nantes, France, 2014.

[12] K. Takahashi, J. Igel, H. Preetz, and S. Kuroda, "Basics and application of Ground-Penetrating Radar as a tool for monitoring irrigation process", in Problems, Perspectives and Challenges of Agricultural Water Management, M. Kumar, Ed. InTech, 2012, pp. 155-180.

[13] A. P. Annan, Ground Penetrating Radar Principles, Procedures Applications. Sensors \& Software Inc., 2003.

[14] Z.-Q. Zhu, L.-X. Peng, G.-Y. Lu, and S.-W. Mi, "Borehole-GPR numerical simulation of full wave field based on convolutional perfect matched layer boundary", J. Cent. South Univ., vol. 20, no. 3, pp. 764-769, 2013 (doi: 10.1007/s11771-013-1546-3).

[15] S. Lambot, F. André, E. Slob, and H. Vereecken, "Effect of antennamedium coupling in the analysis of ground-penetrating radar data", Near Surface Geophys., vol. 10, pp. 631-639, 2012.

[16] W. Kang et al., "A Study of antenna configuration for bistatic Ground-Penetrating Radar", in Proc. of the 16th Int. Conf. of Ground Penetrating Radar (GPR), Hong Kong, China, 2016.

[17] H. V. Baghdasaryan, "Method of backward calculation", in Photonic Devices for Telecommunications: How to Model and Measure, G. Guekos, Ed. Springer, 1999, pp. 56-65.

[18] H. V. Baghdasaryan and T. M. Knyazyan, "Problem of plane EM wave self-action in multilayer structure: An exact solution", Optical and Quant. Electron., vol. 31, pp. 105-1072, 1999.

[19] H. V. Baghdasaryan and T. M. Knyazyan, "Modelling of strongly nonlinear sinusoidal Bragg gratings by the Method of Single Expression", Optical and Quant. Electron., vol. 32, no. 6-8, pp. 869-883, 2000.

[20] H. V. Baghdasaryan and T. M. Knyazyan, "Method of single expression - an exact solution for wavelength scale 1D photonic structures' computer modeling", Proceedings of SPIE, vol. 5260, pp. 141-148, 2003.

[21] H. V. Baghdasaryan, Basics of the Method of Single Expression: New Approach for Solving Boundary Problems in Classical Electrodynamics. Yerevan: Chartaraget, 2013.

[22] H. V. Baghdasaryan, T. M. Knyazyan, T. T. Hovhannisyan, M. Marciniak, and L. Pajewski, "Numerical modelling of GPR groundmatching enhancement by a chirped multilayer structure - output of cooperation within COST Action TU1208", EGU General Assembly 2016, Geophysical Research Abstracts, vol. 18, EGU2016-18507, 2016.

[23] Y.-Y. Li, K. Zhao, J.-H. Ren, Y.-L. Ding, and L.-L. Wu, "Analysis of the dielectric constant of Saline-Alkali soils and the effect on radar backscattering coefficient: A Case Study of Soda Alkaline Saline Soils in Western Jilin Province Using RADARSAT-2 Data", The Scient. World J., vol. 2014, Article ID 563015, 2014.

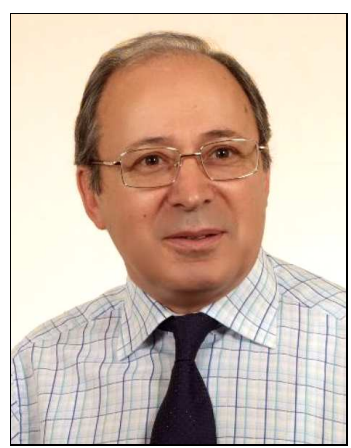

$3 / 2017$
Hovik V. Baghdasaryan graduated in Radiophysics from the Yerevan State University in Yerevan, Armenia, in 1971. He received the Ph.D. degree in Physics and Mathematics from the Institute of Radiophysics and Electronics of the Academy of Sciences of Armenia, in 1980. From 1976 to 1982 , he was with the Institute of Ra- 
diophysics and Electronics of the Academy of Sciences of Armenia as a Junior Research Scientist. From 1983 to 1994, he was with the Research Institute "Lazerayin Tekhnika", Yerevan, Armenia as a Senior Research Scientist. From 1995 to 2016, he was an Associate Professor of Faculty of Radioengineering and Communication Systems at the State Engineering University of Armenia (now National Polytechnic University of Armenia), Yerevan, Armenia. From 2016 he is a Professor at the same Faculty. He is also a Head of Fiber Optics Communication Laboratory of the same university, where his scientific efforts have included work on developing a new nontraditional method of boundary problems solution, called Method of Single Expression (MSE). His main research interests include theory of electromagnetic wave interaction with nonlinear and laser-active media, wave propagation through multilayer and modulated structures, semiconductor and dye laser systems simulation, plasmonics, integrated optoelectronics, fiber optics communication and communication technology. He is a member of OSA. Since 1995 he has been involved in European programmes under different COST Actions. He is a member of the COST Actions TU 1208, MP1403 and MP1401. He is a scientific committee member of International Conference on Transparent Optical Networks (ICTON). He has contributed to a number of national and international conferences and published more than 125 papers in scientific journals and conference proceedings.

E-mail: hovik@seua.am; hovik_b@yahoo.com

National Polytechnic University of Armenia

105 Terian St

0009 Yerevan, Armenia

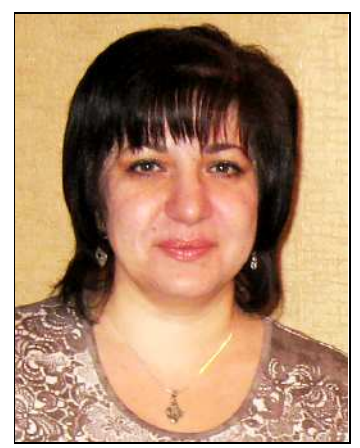

Tamara M. Knyazyan graduated in Radio-engineering from the Yerevan Polytechnic Institute in Yerevan, Armenia, in 1988. She received the M.Sc. degree in communication means from the State Engineering University of Armenia (now National Polytechnic University of Armenia) in Yerevan, Armenia in 1995, and the Ph.D. degree in Radiophysics from the Yerevan State University in Yerevan, Armenia, in 2000. Her postgraduate research dealt with computer modelling of narrowband nonlinear optical filters for DWDM systems by using the Method of Single Expression (MSE). Since 1995, she has been with the Fiber Optics Communication Laboratory at National Polytechnic University of Armenia, as a Research Scientist, where she is involved in the research works on computer modeling of passive and active multilayer and modulated photonic structures. She joined the Faculty of Radioengineering and Communication Systems at National Polytechnic University of Armenia in 1998 as a Senior Lecturer. From 2005 she is an Associate Professor at the same
Faculty. From 2010 to 2015 she was a Head of Chair of Communication Systems at the same Faculty. Since 1995 she has been involved in European programmes under different COST Actions. She is a member of the COST Actions TU 1208, MP1403 and MP1401. Her main research interests include theoretical investigation of electromagnetic waves interaction with $1 \mathrm{D}$ multilayer and modulated photonic structures (including intensity dependent), photonics, plasmonics and modelling of fiber optics communication components. She has contributed to a number of national and international conferences and published more than 100 papers in scientific journals and conference proceedings.

E-mail: ktamara@seua.am; tamknyazyan@yahoo.com National Polytechnic University of Armenia

105 Terian St 0009 Yerevan, Armenia

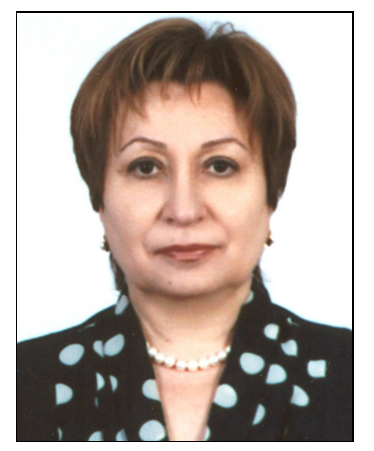

Tamara T. Hovhannisyan graduated in Chemistry from the Yerevan State University, Armenia, in 1976. From 1976 till 1998 she was with ReaKhRom IREA Armenia, as a Scientific Researcher. Since 1998 she has been with Fiber Optics Communication Laboratory at the National Polytechnic University of Armenia as a Research Scientist. Her scientific interests are directed to the numerical analysis of electromagnetic waves interaction with different wavelength-scale multilayer and modulated structures. She has contributed to a number of national and international conferences and published more than 20 papers in scientific journals and conference proceedings. She is member of the COST Actions TU 1208, MP1403 and MP1401.

E-mail: tomi_mark@yahoo.com

National Polytechnic University of Armenia

105 Terian St

0009 Yerevan, Armenia

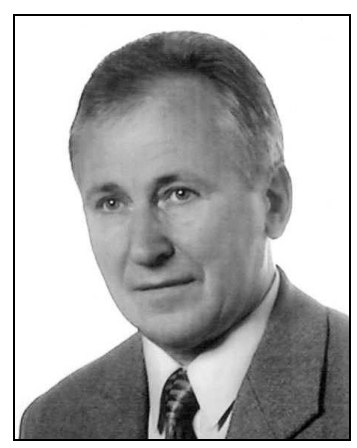

Marian Marciniak graduated in Solid State Physics from Marie Curie-Skłodowska University in Lublin, Poland, in 1977. He holds a Ph.D. degree in Optoelectronics (1989), and a Doctor of Sciences (Habilitation) degree in Physics/ Optics (1997). He is a Professor at the National Institute of Telecommunications, Warsaw, Poland. He has authored over 400 publications, including a number of invited conference presentations. He serves as a Honorary International Advisor to the George Green Institute for Electromagnetics Research, University of 
Nottingham, UK. He is the leading organizer of the International Conferences on Transparent Optical Networks ICTON series since 1999. He serves, together with Prof. Trevor Benson and Prof. Yaping Zhang, as The Editor-in-Chief of Optical and Quantum Electronics journal. He served as the Chairman of COST Action MP0702 "Towards Functional Sub-Wavelength Photonic Structures" (2008-2012). He is a Management Committee Member and the Short-Term Scientific Mission Manager of COST Action TU1208 "Civil Engineering Applications of Ground Penetrating Radar".

E-mail: M.Marciniak@itl.waw.pl; marian.marciniak@ieee.org

National Institute of Telecommunications

1 Szachowa St

04-894 Warsaw, Poland

Lara Pajewski - for biography, see this issue, p. 29. 